\title{
The Economic Dispatch of Cogeneration Systems in the Deregulation Market
}

\author{
Ming-Tang Tsai ${ }^{1, *}$, Guan-Zhi Wang ${ }^{1}$, Chi-Chun Lo ${ }^{2}$ \\ ${ }^{1}$ Department of Electrical Engineering, Cheng-Shiu University, Taiwan \\ ${ }^{2}$ Department of Engineering and Maintenance, Chang Cung Memorial Hospital, Taiwan
}

Copyright (C) 2015 by authors, all rights reserved. Authors agree that this article remains permanently open access under the terms of the Creative Commons Attribution License 4.0 International License.

\begin{abstract}
This paper presents an Improved Genetic Algorithm (IGA) to solve the economic dispatch of cogeneration systems in a deregulated market. Based on bi-lateral trade between cogeneration systems and utility, an objective function is derived for the cogeneration systems to obtain the maximal profit while stratifying the operational constraints. IGA takes advantage of the conventional GA and tabu search. The crossover and mutation schemes of conventional GA are improved by a competition mechanism to avoid the prematurity. Tabu Lists with heuristic rules are also employed in the searching process to enhance performance. The IGA approach is demonstrated by using a real cogeneration system. Results can offer an efficient way for cogeneration systems to promote the competed ability.
\end{abstract}

Keywords Deregulated Market, Economic Dispatch, Cogeneration Systems, Genetic Algorithm

\section{Introduction}

In the recent year, cogeneration systems which are known the simultaneous production of electricity and steam had now been extensively utilized by the industries. It offers a reliable, efficient, and economic mean to supply the both thermal steam and electric power. The electric power generated and thermal steam from the cogeneration systems can be transmitted to buyer. The cogeneration systems also brought the electricity power from utilities when the electricity price is lower than the marginal cost of electricity produce. Some efficient strategies had been developed to minimize their operation cost in the dispatch schedules[1-3]. In the competitive market, these papers did not involve the deregulated problems. From the view of cogeneration systems, the operating strategy determines the optimal distribution among the in-plant generation, bi-lateral contract trade, steam sold, and electricity brought/sold while satisfying the overall system constraints.

It is complicated to perform the economic dispatch of cogeneration systems, especially considering the best strategy in a deregulated market. Conventional methods become more difficult to solve. Recently, some artificial intelligence (AI) algorithms had been developed in [4-6]. Solution strategies proposed by AI need to consider a large solution space. Conventional methods may be faster, they are very often limited by the problem structure and may diverge to a local minimum. This paper presents an Improved Genetic Algorithm (IGA) based Genetic Algorithms[7] to solve the economic dispatch of cogeneration systems.

GA is based on the operation of human immune system. The immune system is a basic bio-defense system against viruses and disease-causing organisms. This complex defense mechanism combines genes to deal with the inbreaking antigens. Using this heuristic algorithm, this paper proposed IGA which further improves GA. In order to avoid prematurity, the crossover and mutation mechanism is refined by a competition and auto-adjust scheme. Crossover and mutation were combined in one step in IGA, and a competition mechanism was implemented to automatically determine the choice of either one. Numerical examples are also provided to show its effectiveness.

\section{Problem Formulation}

The proposed algorithm was tested on a cogeneration system which has $\mathrm{k}$ back-pressure turbine and $\mathrm{n}$ extraction condenser steam turbine for generating power as shown in Fig.1[2]. Models determination for optimal operation evaluation, such as the $\mathrm{I} / \mathrm{O}$ curve of boiler and turbine are included in this paper 


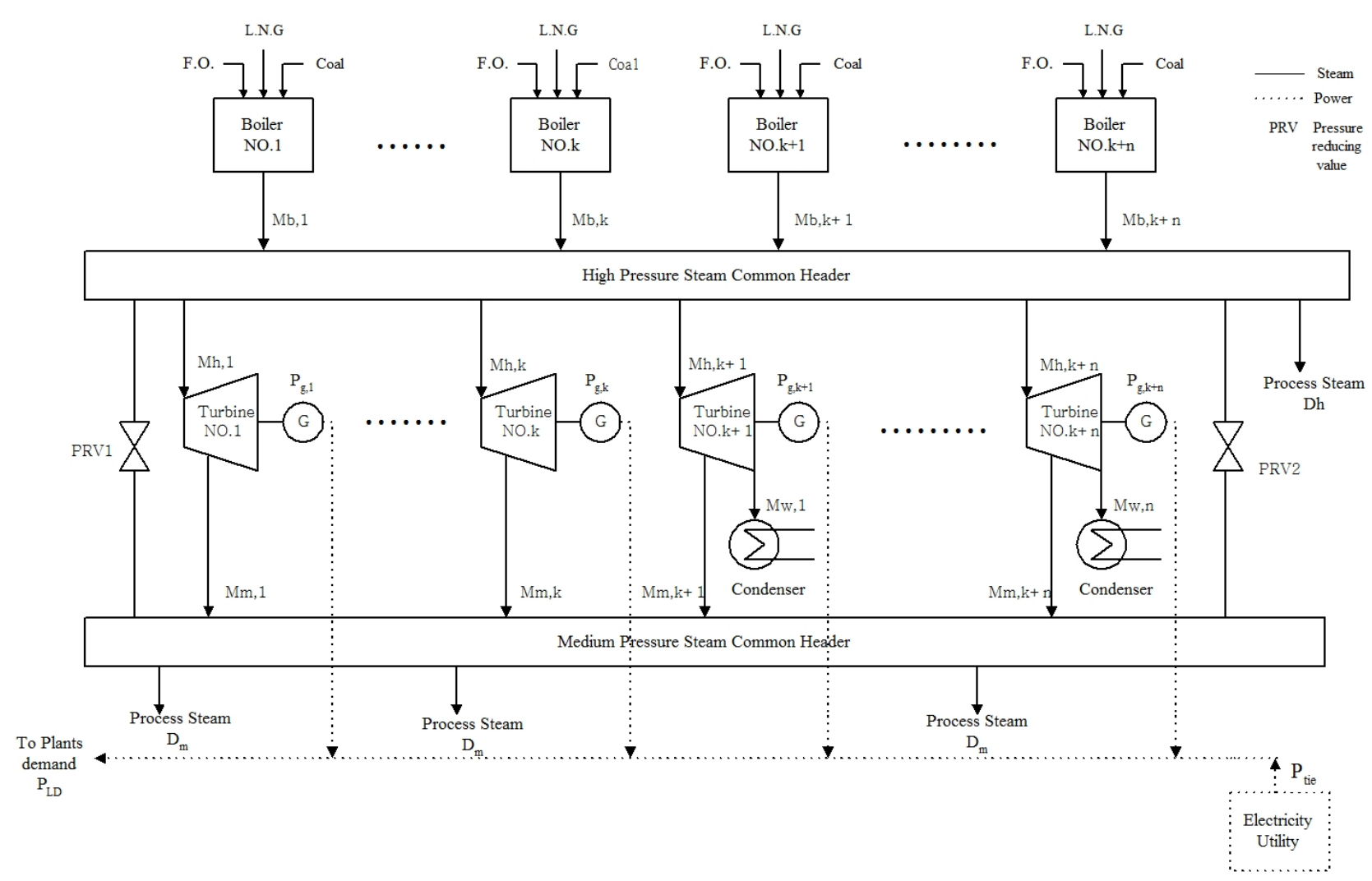

Figure 1. The diagram of the cogeneration system

\subsection{I/O Cost Curve of Boilers}

It is assumed that the $\mathrm{I} / \mathrm{O}$ curve of a boiler is a 3 -rd order polynomial, we have

$\mathrm{F}_{\mathrm{bi}}\left(\mathrm{M}_{\mathrm{bi}}(\mathrm{t})\right)=\mathrm{A}_{\mathrm{o}}+\mathrm{A}_{1} \times \mathrm{M}_{\mathrm{bi}}(\mathrm{t})+\mathrm{A}_{2} \times \mathrm{M}_{\mathrm{bi}}^{2}(\mathrm{t})+\mathrm{A}_{3} \times \mathrm{M}_{\mathrm{bi}}^{3}(\mathrm{t})$

$\mathrm{i}=1,2, \ldots, \mathrm{k}+\mathrm{n}$

$F_{b i}\left(M_{b i}\right)$ :Enthalpy of the $i$-th boiler at $i$-th bus (MBTU/H).

$M_{b i}$ :Steam output of the $\boldsymbol{i}$-th boiler at $\boldsymbol{i}$-th bus(T/H).

$A_{o}, A_{1}, A_{2}, A_{3}:$ Coefficients of the $\mathrm{I} / \mathrm{O}$ operation curve.

\subsection{I/O Operation Curve of Steam Turbines}

For back-pressure turbine generator, the power equation for turbine $i$ can be formulated by

$$
\begin{aligned}
& \mathrm{P}_{\mathrm{gi}}(\mathrm{t})=\mathrm{K}_{\mathrm{oi}}+\mathrm{K}_{1 \mathrm{i}} \mathrm{M}_{\mathrm{mi}}(\mathrm{t})+\mathrm{K}_{2 \mathrm{i}} \mathrm{M}_{\mathrm{mi}}^{2}(\mathrm{t}) \\
& \mathrm{i}=1,2, \ldots ., \mathrm{k}
\end{aligned}
$$

For extraction-condense turbine generator, the power equation for turbine $i$ can be written by

$$
\begin{aligned}
& \mathrm{P}_{\mathrm{gi}}(\mathrm{t})=\mathrm{K}_{\mathrm{oi}}+\mathrm{K}_{1 \mathrm{i}} \mathrm{M}_{\mathrm{mi}}(\mathrm{t})+\mathrm{K}_{2 \mathrm{i}} \mathrm{M}_{\mathrm{wi}}(\mathrm{t}) \\
& \mathrm{i}=1,2, \ldots, \mathrm{n}
\end{aligned}
$$

where $M_{m i}$ is the medium pressure extraction flow of $i$ turbine. $P_{g i}$ is the generated electric power from turbine $\boldsymbol{i} ; K_{o i}, K_{1 i}, K_{2 i}$ are coefficients of turbine $\boldsymbol{i}$ which can then be found by curve fitting technique with field data.

\subsection{Objective Function and Constraints}

The objective function is to determine the economic dispatch of cogeneration system for maximizing the profit by considering the bi-lateral contracts and operational constraints. The objective function including fuel cost and interchange cost with utility is formulated as

\section{Maximize SBCE+ESCE+ESSE-EBCE-EBSE- -WC-FC-OC}

(1) $\boldsymbol{S B C E}$ is the steam sold benefit by contract exchanged, which is formulated as

$$
S B C E=D_{m} \times B C_{m}+D_{h} \times B C_{h}
$$

(2) $\boldsymbol{E S C E}$ is the electricity sold benefit with contract exchanged, which is formulated as

$$
\begin{aligned}
& E S C E=P_{\text {inside }} \times B C_{c_{-} \text {inside }}+ \\
& +P_{s c_{-} \text {outside }} \times B C_{s c_{\text {_outside }}}
\end{aligned}
$$

(3) $\boldsymbol{E S S E}$ is the electricity sold benefit in a spot market, which is formulated as[8]

$$
E S S E=a_{1} \times P_{S S_{\_} \text {outside }}^{2}+b_{1} \times P_{S S_{\_} \text {outside }}+c_{1}
$$

(4) $\boldsymbol{E} \boldsymbol{B C} \boldsymbol{E}$ is the electricity brought cost with contract exchanged, which is formulated as 


$$
E B C E=P_{b c \_o u t s i d e} \times B C_{b c \_o u t s i d e}
$$

(5) $\boldsymbol{E B S E}$ is the electricity sold cost in a spot market, which is formulated as[8]

$$
E B S E=a_{2} \times P_{b s_{-} \text {outside }}^{2}+b_{2} \times P_{b s_{-} \text {outside }}+c_{2}
$$

(6) $\boldsymbol{W C}$ is the wheeling cost with utility, which is formulated as

$$
\begin{aligned}
& W C=\left[P_{\text {inside }}+P_{s c_{-} \text {outside }}+P_{s s_{\_} \text {outside }}+\right. \\
& \left.+P_{b c_{\_} \text {outside }}+P_{b s_{-} \text {outside }}\right] \times K
\end{aligned}
$$

(7) $\boldsymbol{F C}$ is the fuel cost, which is formulated as

$$
F C=\sum_{i=1}^{k+n} F_{b i}\left(M_{b i}\right) * B C_{-} \text {oil }
$$

(8) $\boldsymbol{O C}$ includes maintain cost, management cost, discount cost, water cost,...etc.(NT\$).

$D_{m}:$ The medium pressure steam demand (T)

$B C_{m}:$ The price of medium pressure steam(NT\$/T)

$D_{h}:$ The high pressure steam demand (T)

$B C_{h}$ : The price of high pressure steam(NT\$/T)

$P_{\text {inside }}$ : The load demand of industry(MW)

$B C_{C_{\text {_ }} \text { inside }}:$ The price of contract electricity with industry (NT\$/MW)

$P_{\text {Sc_outside }}:$ The contract electricity with utility (MW)

$B C_{S C_{\text {_outside }}}:$ The price of contract electricity with utility(NT\$/MW)

$P_{\text {SS_outside }}:$ The expected electricity(MW)

$P_{b c \_ \text {outside }}:$ The contract electricity with utility(MW)

$B C_{b c_{-} \text {outside }}:$ The price of contract electricity with utility(NT\$MW)

$P_{b s \_o u t s i d e}:$ The expected electricity(MW)

$\mathrm{K}:$ The wheeling $\operatorname{cost}(\mathrm{NT} \$ \mathrm{MW})$

$B C \_$oil : The fuel price (NT\$/MBTU-H)

The constraints considered are described as follows:

- Steam balance for boilers, turbine and industrial process are

$$
\sum_{i=1}^{k+n} M_{b i}-D_{h}-\sum_{i=1}^{k+n} M_{h i}=0
$$

$$
\begin{gathered}
\sum_{i=1}^{k+n} M_{h i}-\sum_{i=1}^{k+n} M_{m i}-\sum_{i=1}^{k+n} M_{w i}=0 \\
\sum_{i=1}^{k+n} M_{m i}-D_{m}=0
\end{gathered}
$$

where $M_{h i}$ is the high pressure injection flows of turbine $i$-th bus. $D_{h i}$ and $D_{m i}$ are the high and medium pressuresteam demands at $i$-th bus.

- Power balance in the power system

$$
\begin{gathered}
\sum_{i=1}^{k+n} P_{g i}-P_{\text {inside }}-P_{s c \_o u t s i d e}+P_{s s \_o u t s i d e} \\
+P_{b c \_o u t s i d e}+P_{b s \_o u t s i d e}=0
\end{gathered}
$$

- Operation constraints for boilers, steam turbine, power generation, emission control:

$$
\begin{gathered}
M_{b i}^{\min } \leq M_{b i} \leq M_{b i}^{\max } \\
M_{h i}^{\text {min }} \leq M_{h i} \leq M_{h i}^{\max } \\
M_{m i}^{\text {min }} \leq M_{m i} \leq M_{m i}^{\max } \\
P_{g i}^{\min } \leq P_{g i} \leq P_{g i}^{\max }
\end{gathered}
$$

$M_{b i}^{\min }, M_{b i}^{\max }$ :Lower and upper limits of flow for boiler $\boldsymbol{i}$.

$M_{h i}^{\min } M_{h i}^{\max }$ :Lower and upper limits of high pressure injection flows for turbine $\boldsymbol{i}$.

$M_{m i j}^{\min } M_{m i j}^{\max }$ :Lower and upper limits of medium pressure extraction flows for turbine $\boldsymbol{i}$.

$P_{g i j}^{\min } P_{g i j}^{\max }$ : Lower and upper limits of the generated electric power for turbine $i$.

\section{Solution Algorithm}

To enhance the performance of GA, an improved GA (IGA) was developed as follows.

\subsection{Encoding}

The gene is encoded as a chromosome string which produced by equation (20). If the GA search is terminated, the chromosome will then be decoded.

$$
\begin{gathered}
\left.D 2 B\left\{\left[\left(M_{i}-M_{i}^{\text {min }}\right)\right) / \text { resol }_{i}\right\rceil\right\}, \\
\text { resol }_{i}=\left(M_{i}^{\text {max }}-M_{i}^{\text {min }}\right) /\left(2^{\text {bit }}-1\right)
\end{gathered}
$$


D2B: decimal to binary conversion

bit : the number of bits in a chromosome

\subsection{Fitness Function Evaluation}

The fitness score of each gene is obtained by calculating the objective function and taking (12) (19) into account. If one or more variables violate their limits, the corresponding chromosome will be put into the tabu list to avoid generating the same infeasible solution again.

\subsection{Offspring}

The offsprings are the new chromosomes obtained from crossover and mutation. Crossover is a structured recombination operation by exchanging genes of two parents. Mutation is the occasional random alteration of genes. An improved crossover and mutation (ICM) scheme is used in this paper. The ICM procedure can be described as follows.

(i) Randomly select two parents, and generate offsprings by introducing $\mathrm{C}(\mathrm{g})$ with

(a) If rand $<\mathrm{C}$ (g) : use mutation; (b)If rand $>\mathrm{C}$ (g) : use crossover.

The parameters for crossover and mutation are 0.2 and 0.02 , respectively.

rand : the uniform random number in $(0,1)$,

$\mathrm{C}$ : the control parameter with initial value set to 0.5 , $0 \leq \mathrm{C} \leq 1$,

$\mathrm{g}$ : the current generation number.

(ii) If $F_{\min }(g-1)>F_{\min }(g)$ comes from crossover, the control parameter $\mathrm{C}(\mathrm{g}+1)$ will decrease. We have

$$
C(g+1)=C(g)-\frac{T_{1}}{g_{\max }}
$$

where $T_{1}$ is the regulating factor, and $g_{\max }$ is the maximum generation number.

(iii) If $F_{\min }(g-1)>F_{\min }(g)$ comes from mutation, $\mathrm{C}(\mathrm{g}+1)$ will increase. For $F_{\min }(g-1)>F_{\min }(g)$, we have

$$
C(g+1)=C(g)+\frac{T_{1}}{g_{\max }}
$$

(iv) If $F_{\min }(g-1) \leq F_{\min }(g)$, the control parameter needs to hold back.

$$
\begin{gathered}
\text { If } \mathrm{C}(\mathrm{g})>\mathrm{C}(\mathrm{g}-1), C(g+1)=C(g)-\frac{T_{2}}{g_{\max }} \\
\text { Else, if } \mathrm{C}(\mathrm{g}) \leqq \mathrm{C}(\mathrm{g}-1), C(g+1)=C(g)+\frac{T_{2}}{g_{\max }}
\end{gathered}
$$

\subsection{Tabu List[9]}

A tabu list will be constructed to define forbidden moves, such as (i) the solutions just visited except the best solution in the current generation,

(ii) the local optimum ever visited,

(iii) the chromosomes violating constraints,

(iv) the solution space cannot accord with bargain condition.

\subsection{Elitism Selection}

The $2 p$ chromosomes are then ranked in ascending order according to their fitness values. "b" individuals with the best fitness are kept as the parents for the next generation. Other individuals in the combined population of size (2p-b) have to compete by adopting the roulette wheel approach to get selected in the next generation.

\subsection{Stopping Rule}

The process of generating new trials with the best fitness will be continued until the fitness values are optimized or the maximum generation number is reached.

\section{Case Study}

The proposed algorithm was tested on a practical cogeneration as shown in Fig. 1. It contains 5 back-pressure steam turbine, 2 extraction condenser steam turbine, and 7 steam boilers. The demands of high-pressure steam and medium-pressure steam are $D_{h}=621 \mathrm{~T} / \mathrm{H}$ and $D_{m}=50 T / H$, respectively. The steam price for high-pressure steam and medium-pressure steam is $485 \mathrm{NT} \$ / \mathrm{T}$ and $970 \mathrm{NT} \$ / \mathrm{T}$. The contract electricity solid and the contract electricity bought are 3400NT\$/MW and $2100 \mathrm{NT} \$ \mathrm{MW}$. The unit wheeling cost is $100 \mathrm{NT} \$ / \mathrm{MW}$. The detail data of this cogeneration system is listed in [10]. Each case is written with Matlab language on a Person Computer with Intel i5-4 core and 4G DDRAM.

\section{(a) Condition 1: The Electricity is Traded by Spot Market $\left(P_{\text {inside }}=65 \mathrm{MW}, 65 \mathrm{MW}, 70 \mathrm{MW}\right)$}

Fig. 2 shows the convergent characteristics for economic dispatch of cogeneration systems. The stop rule for IGA is set 300 generations. From the Fig. 2, due to the industrial $\operatorname{load}\left(P_{\text {inside }}\right)$ is increased from $60 \mathrm{MW}$ to $70 \mathrm{WM}$, the cogeneration systems will sell more electricity to spot market and the profit is thus increase. The maximal profits for $60 \mathrm{MW}, 65 \mathrm{MW}$, and $70 \mathrm{MW}$ load are $212103 \mathrm{NT} \$ / \mathrm{H}$, $217487 \mathrm{NT} \$ / \mathrm{H}$, and 221918NT\$/H.

\section{(b) Condition 2: The Cogeneration Systems Have the Various Contracts with Utility}

Table 1 shows the profits of the various loads and contracts. From the Table 1, the contract is more, the profit will be decreased. It is shown that the sell/buy expected curve will provide the various competitive models and the 
profit is relative with electricity sold. If the load is larger and contract is less, the cogeneration systems can buy the electricity from utility and sell the electricity to industry for obtaining their maximal profit. Fig. 3 is the convergent characteristics of IGA in all tests. The stop rule is also set 300 generations.

Table 1. The profits of the various loads (unit:NT\$/H)

\begin{tabular}{|c|c|c|c|}
\hline Contracts & 80(MW) & 90(MW) & $100(\mathrm{MW})$ \\
\hline 15(MW) & 197002 & 231814 & 247547 \\
\hline 20(MW) & 184352 & 190125 & 212553 \\
\hline 25(MW) & 170263 & 189685 & 201123 \\
\hline
\end{tabular}

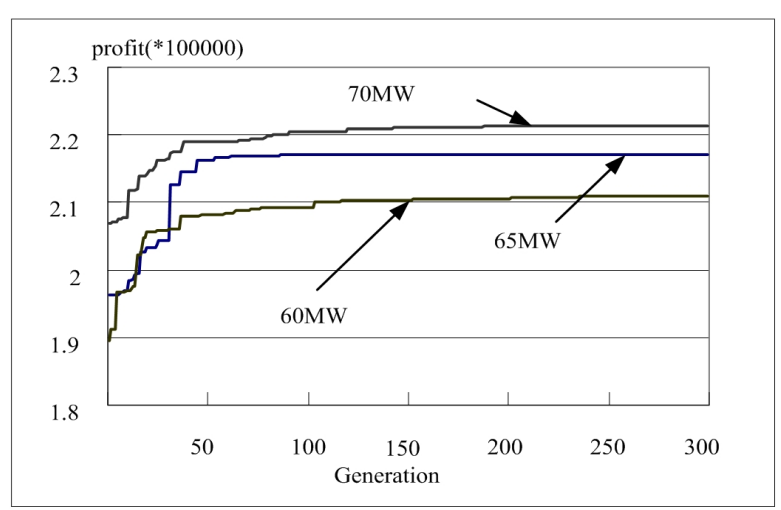

Figure 2. The convergent characteristics of IGA

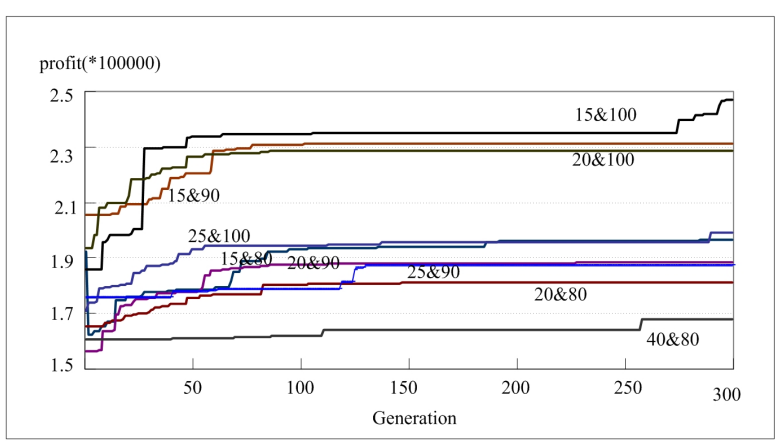

Figure 3. The convergent characteristics of IGA

\section{(c) Robust Test}

Table 2 shows the robust tests of GA and IGA. From the Table 2, it is shown that IGA can get a better value than GA. The optimum improvement of IGA is over GA.

Table 2. The robust tests of GA and IGA Unit:NT\$/H

\begin{tabular}{|c|c|c|c|}
\hline Item Load(Pinside) & $60(\mathrm{MW})$ & $65(\mathrm{MW})$ & $70(\mathrm{MW})$ \\
\hline IGA & 212103 & 217487 & 221918 \\
\hline GA & 207190 & 211430 & 219030 \\
\hline Deviation & 4913 & 6057 & 2888 \\
\hline
\end{tabular}

\section{Conclusions}

This paper presents an IGA to solve the economic dispatch of cogeneration systems in a competitive market. The effectiveness of IGA has been demonstrated by numerical examples. IGA is superior to GA in two ways: one is the use of tabu lists to avoid invalid searches, especially in applications with many constraints and local optimum; another one is the automatic regulation of the frequency of crossover and mutation operations, particularly in applications sensitive to the probabilistic rates. IGA has great potential to be further applied to many ill-conditioned problems in power system planning and operations.

\section{Acknowledgements}

Financial support for this work by the Ministry of Science and Technology in R.O.C. under contract number NSC101-2221-E-230-021 is gratefully appreciated.

\section{REFERENCES}

[1] Çakir, U, Çomakli, K., and Yükse, F., 2012. The role of cogeneration systems in sustainability of energy. Energy Conversion and Management, 63(1), 196-202.

[2] Tsay, M.T., Lin, W.M., and Lee, J.L., 2001. Application of evolutionary programming for economic dispatch of cogeneration systems under emission constraints. International Journal of Electrical Power \& Energy Systems, 23(8), 805-812.

[3] Daniel, S., Lou, C., K., and Min, J., 2013. Economic models for cogeneration facilities and host utilities under the right to sell provision. Electric Power Systems Research, 103, 214-222.

[4] Jong, Y.Y., and Li, C.Y., 2002. Genetic Algorithms Based Economic Dispatch for Generation Units Considering Multiplant Multibuyer Wheeling. IEEE Transactions on Power Systems, 17(1), 134-140.

[5] Chen. S.L., Tsay, M.T., and Gow, H.J., 2005. Scheduling of cogeneration plants considering electricity wheeling using enhanced immune algorithm. International Journal of Electrical Power \& Energy Systems, 27(1), 31-38.

[6] Basu, 2012. Artificial immune system for combined heat and power economic dispatch. International Journal of Electrical Power \& Energy Systems, 43(1), 1-5.

[7] Chang, H.H., 20141. Genetic algorithms and non-intrusive energy management system based economic dispatch for cogeneration units. Energy, 36(1), 181-190.

[8] Krnav, J., and Grald, B.S., 1998. Auction Market Simulator for Price Based Operation. IEEE Transactions on Power Systems, 13(1), 250-255

[9] Glover, F., 1990. Tabu search-part II. ORSA J. Comput., 1990, 2(1), 4-32.

[10] Lee, J.L., 1999. Application of evolutionary programming to optimal dispatch of cogeneration system. The Master Thesis of National Sun Yat-Sen University. 